\title{
Effect of ion beam irradiation on metal particle doped polymer composites
}

\author{
N L SINGH*, SEJAL SHAH, ANJUM QURESHI, A TRIPATHI ${ }^{\dagger}$, F SINGH ${ }^{\dagger}$, D K AVASTHI \\ and P M RAOLE ${ }^{\dagger \dagger}$ \\ Department of Physics, M. S. University of Baroda, Vadodara 390 002, India \\ ${ }^{\dagger}$ Inter University Accelerator Centre, Aruna Asaf Ali Marg, New Delhi 110 067, India \\ ${ }^{\dagger}$ FCIPT, Institute for Plasma Research, Gandhinagar 382 044, India
}

MS received 14 August 2009; revised 14 October 2009

\begin{abstract}
Polymethyl methacrylate (PMMA) was prepared by solution polymerization method. Different concentrations (10, 20 and 40\%) of $\mathrm{Ni}$ powder were dispersed in PMMA and the composite films were prepared by casting method. These films were irradiated with $120 \mathrm{MeV} \mathrm{Ni}{ }^{10+}$ ions at a fluence of $5 \times 10^{12}$ ions $/ \mathrm{cm}^{2}$. Electrical, structural and chemical properties of the composites were studied by means of an LCR meter, X-ray diffraction, FTIR spectroscopy and SEM/AFM, respectively. The results showed that the conductivity increases with metal concentration and also with ion beam irradiation. This reveals that ion beam irradiation promotes the metal/polymer bonding and converts polymeric structure into hydrogen depleted carbon network. It was observed from XRD analysis that percentage crystallinity and crystalline size decrease upon irradiation. This might be attributed to rupture of some polymeric bonds, which is also corroborated with FTIR spectroscopic analysis. Ion beam tempts graphitization of polymeric material by emission of hydrogen and/or other volatile gases. Surface morphology of the pristine and irradiated films was studied by atomic force microscopy (AFM)/scanning electron microscopy (SEM). Result showed that the surface roughness increases after ion beam irradiation.
\end{abstract}

Keywords. Composite materials; ion beam irradiation; dielectric properties; X-ray diffraction.

\section{Introduction}

Various metal fillers were incorporated in polymers to produce novel functionalized composites, which have found extensive applications, such as reinforcement (Creighton and Clyne 2000), conductance in the fuel cell (Blunk et al 2003), electrical heating (Song and Zheng 2004), electromagnetic interference shielding (Verma et al 2003) and positive temperature coefficient (PTC) resistors (Luo et al 1998). It is well known that the performance of composites are closely correlated with the spatial distribution (topology) and connectivity of the fillers (McLachlan et al 1990; Louis and Gokhale 1996). The electrical properties of these composites are directly related to the permittivity and conductivity of the constituent phases, size, shape, and the volume fraction of the filler and the adhesion between metals and polymers (McLachlan et al 1990; Verma et al 2003). The concentration of the conductive inclusions has been proved to be a crucial parameter governing the electrical behaviour of the composites. When the filler content is low, the mean distance between metal particles or clusters is sufficiently large and conductance is restricted by the presence of the dielec-

\footnotetext{
*Author for correspondence (singhnl_msu@yahoo.com)
}

tric matrix. Some other factors, such as preparation method, interface between the fillers and polymers, and possible interactions between the conductive and non conductive phases, are also significant (Zhang et al 2006).

Ion beam technique is a widely used and powerful tool for the modification of different materials including polymers. The growing interest in the ion beam irradiation of polymeric material is due to possibility of verification of the technological parameters as the fundamental reason and also considering some possible applications to device fabrication. Thus, optical, mechanical, electrical and chemical properties can be selectively modified using ion beam bombardment (Solfield et al 1993; Vacik et al 1997). Ion irradiation of polymer is followed by the processes of macromolecular distribution, cross-linking, free radicals formation, carbonization and oxidation (Bedell et al 1990; Hnatowicz et al 1994). Therefore, understanding of certain structural rearrangements of the polymer composites opens a way to design devices with required parameters. However, there are numerous reports in the literature dealing with ion beam induced dielectric analysis, which provides information about important parameters such as permittivity, loss factor and conductivity.

The dielectric parameters are important by themselves but become more significant when correlated to activity on a molecular level since chemical structure, polarity 
and interaction of molecules determine the ability of dipoles to respond to electromagnetic field (Shepard and Twombly 1996; Campbell et al 2001). This correlation allows probing the chemistry, flow and molecular mobility (relaxations) of polymers and composites which is related to processing and morphology of polymer composites (Shepard and Twombly 1996). Moreover, dielectric analysis is also important in the development of electrode and electrolyte materials for solid state electrochemical devices such as batteries, sensors, fuel cells, super capacitors and electrochromics as well as in the development of microelectronic packaging materials for performance optimization of high frequency devices (Raja et al 2004). The aim of the present work is to study the effect of swift heavy ion (SHI) irradiation on structural and dielectric properties of polymer-metal composites.

\section{Experimental}

\subsection{Sample preparation and irradiation}

PMMA $\left(\mathrm{C}_{5} \mathrm{O}_{2} \mathrm{H}_{8}\right)_{n}$ was used as polymer matrix and was synthesized by solution polymerization method (Qureshi et al 2007). Ni metal filler was purchased from Laborchemikalein, India. The concentration of filler was varied as 10, 20 and
$40 \%$ in the composites. Ni filled PMMA composite films were prepared by solution casting method. Toluene: acetone $(50: 40)$ was used as a solvent to dissolve the polymers. The mixture of polymer and filler was stirred with different volume fractions of filler at room temperature using magnetic stirrer at a high speed of around $700 \mathrm{rpm}$ until all polymer grains dissolved. After obtaining a homogeneous mixture, the mixture was poured on to a clean glass trough. The solvent was evaporated at room temperature $\left(25^{\circ} \mathrm{C} \pm 1^{\circ} \mathrm{C}\right)$ to get thin films of polymer composites (thickness, $\sim 70 \mu \mathrm{m}$ ) which were then dried in vacuum oven at $30^{\circ} \mathrm{C}$ (Shah et al 2008). The films were irradiated with $120 \mathrm{MeV} \mathrm{Ni}^{10+}$ beam at a fluence of $5 \times 10^{12}$ ions $/ \mathrm{cm}^{2}$ using 15 UD Pelletron at Inter University Accelerator Centre (IUAC), New Delhi. The samples were irradiated in vacuum of the order of $10^{-6}$ torr at an ambient temperature.

\subsection{Characterization}

Alternating current (a.c.) dielectric properties of the samples were measured using Solartron-1260 Impedance gain/phase analyser in the frequency range $100 \mathrm{~Hz}-10 \mathrm{MHz}$ at room temperature. The electrodes were painted with silver paste before measurement. Conductivity and dielectric permittivity of the samples were calculated using standard formula as reported elsewhere (Shah et al 2008). The structural

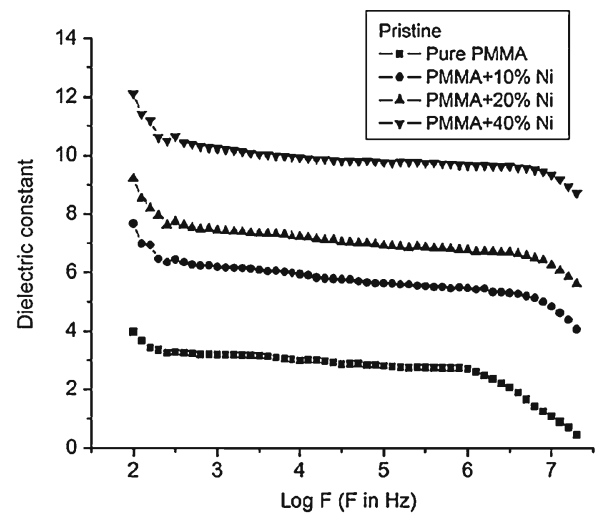

(a)

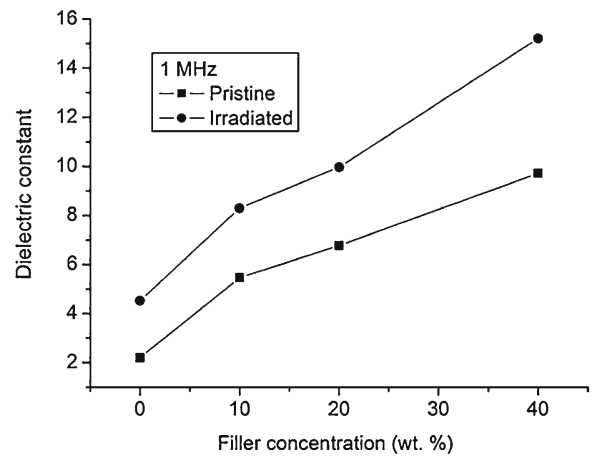

(c)

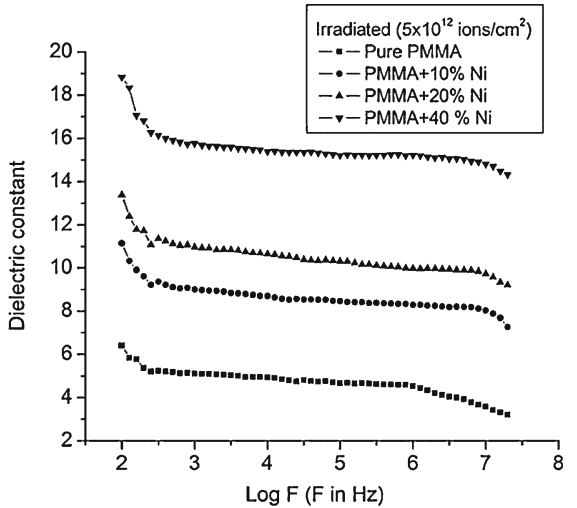

(b)

Figure 1. Dielectric constant vs log frequency for (a) pristine, (b) irradiated samples and (c) dielectric constant vs concentration of filler at $1 \mathrm{MHz}$. 
studies were carried out by X-ray powder diffractometer (model: Shimadzu, XRD-6000) with $\mathrm{CuK}_{\alpha}$ radiation $(1.5418 \AA)$ for a Bragg angle of $35^{\circ}<2 \theta<60^{\circ}$. All measurements were carried out at ambient temperature. FTIR spectra of pristine and irradiated PMMA films were recorded in the wave number range $4000-500 \mathrm{~cm}^{-1}$ using ThermoNicolet (NEXUS 670) FTIR spectrometer with a resolution of $4 \mathrm{~cm}^{-1}$. Surface morphology of pristine and irradiated surfaces was studied by means of scanning electron microscopy (Oxford LEO 440I)/atomic force microscopy (Digital Nanoscope IIIa Instrument Inc.) in tapping mode.

\section{Results and discussion}

\subsection{Dielectric property of composites}

Figure 1(a,b) shows the variation of dielectric constant of pristine and irradiated PMMA-Ni composites at different concentrations of Ni-metal as a function of frequency. The dielectric constant is found to decrease moderately with frequency for pristine and irradiated samples at all concentrations. At low frequencies, the polarization follows the change of the electric field, the loss is minimal and

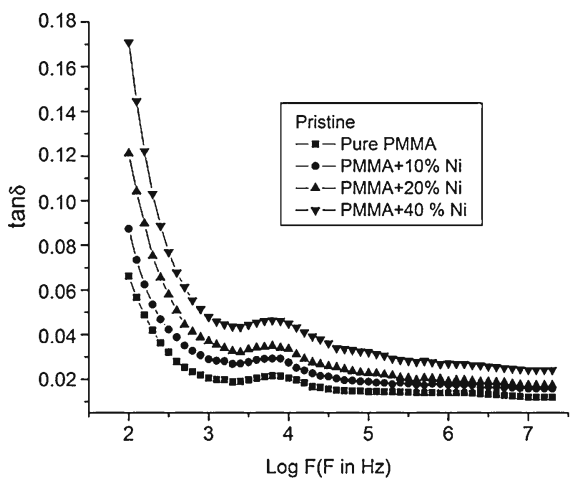

(a)

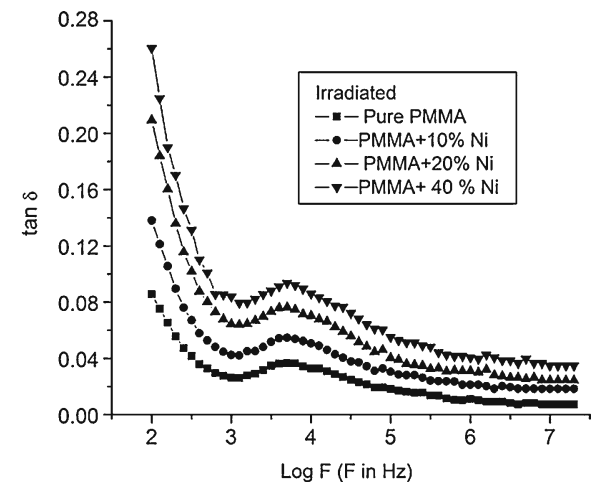

(b)

Figure 2. Dielectric loss vs log frequency for (a) pristine and (b) irradiated films.

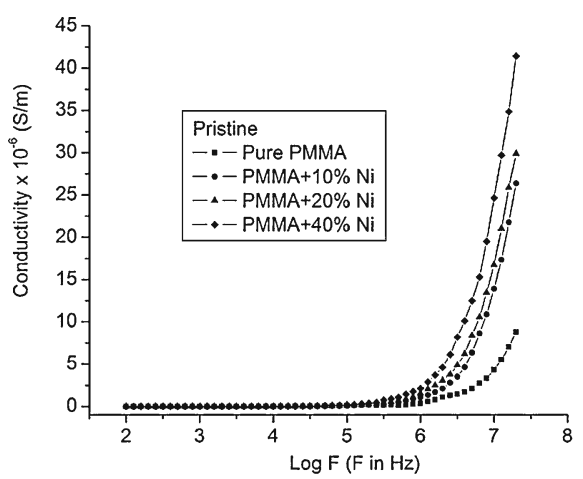

(a)

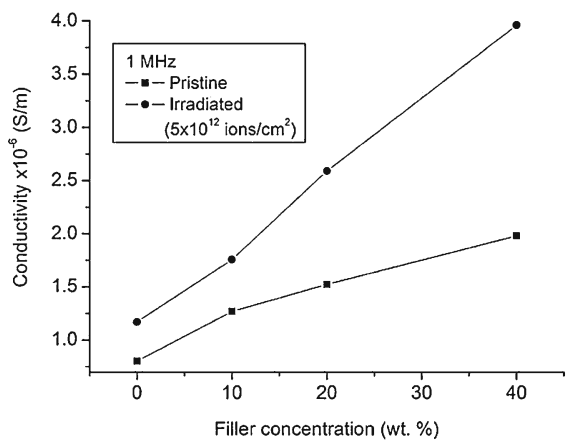

(c)

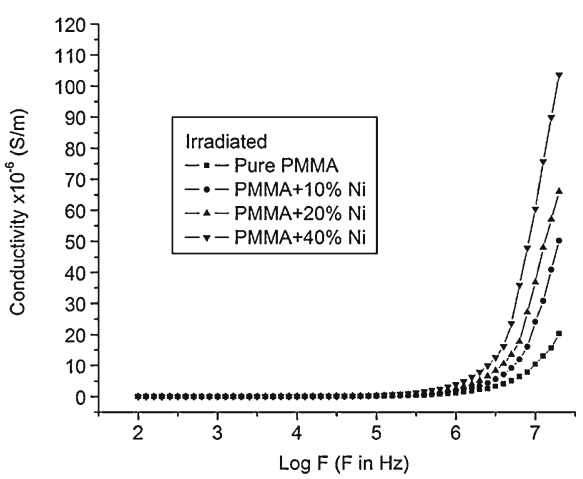

(b)

Figure 3. A.C. conductivity vs log frequency for (a) pristine, (b) irradiated composite films and (c) a.c. conductivity vs filler concentration at $1 \mathrm{MHz}$. 
contribution to the dielectric constant is maximal. At high frequencies, the electric field changes too fast for the polarization effects to appear. The variation of dielectric constant as a function of concentration for pristine and irradiated $\mathrm{Ni}$-PMMA composites at $1 \mathrm{MHz}$ is shown in figure 1(c). The increase in the dielectric constant with filler content is a direct consequence of interfacial polarization effect (Foulger 1999; Ghany et al 2000; Singh et al 2008) between polymer and filler particles. The dielectric constant of the compo-

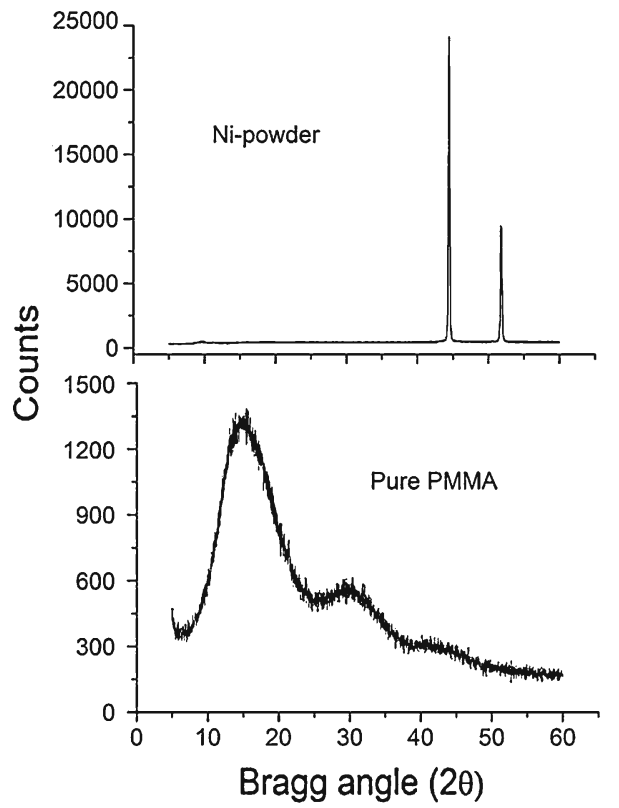

(a)

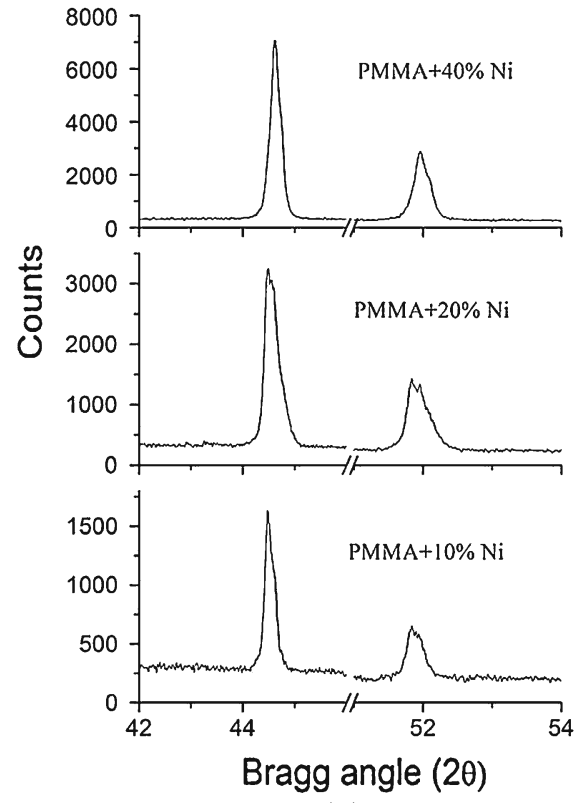

(c) sites is also a function of its capacitance, which is proportional to the quantity of charge stored on either surface of the sample under an applied electric field (Yu et al 2000; Dang et al 2004). In PMMA composite (filled with conducting fillers), the quantity of accumulated charges will increase because of the polarization of PMMA/filler at interfaces. The polarization makes an additional contribution to the charge quantity. From this point of view the dielectric constant of the composites be higher than that of the pure

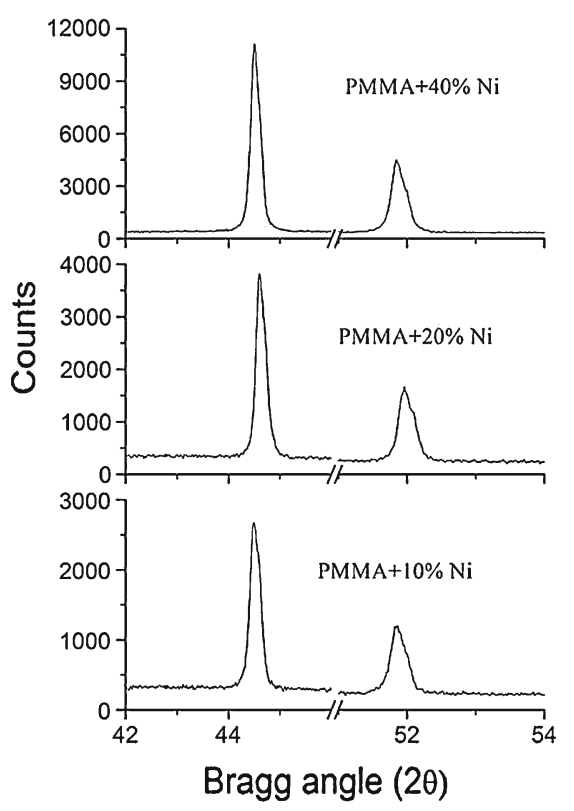

(b)

Figure 4. XRD spectrum of (a) pure PMMA and filler (Ni-powder), (b) pristine and (c) irradiated composite films. 
polymers and it also increases with increasing filler content. The experimental results in figure 1(a) also support this explanation. Therefore, the materials with high dielectric constant could be prepared by adding the conducting fillers. As evident from figure $1(\mathrm{a}, \mathrm{b})$, the dielectric constant remains almost constant up to $100 \mathrm{kHz}$. At these frequencies, the motion of the free charge carriers is constant and so the dielectric constant presumably remains unchanged. As frequency increases further (i.e. beyond $100 \mathrm{kHz}$ ), the charge carriers migrate through the dielectric and get trapped against defect sites and induces an opposite charge in its vicinity. At these frequencies, the polarization of trapped and bound charges cannot take place and hence the dielectric constant decreases (Jonscher 1983). It was also observed that dielectric constant increases after ion beam irradiation. The increase in dielectric constant may be attributed to the chain scission, as a result the increase in the number of free radicals, etc. Figure 2 (a, b) shows frequency dependence of the dielectric loss of the Ni-PMMA composite for pristine and irradiated samples. The dielectric loss decreased exponentially at low frequency and then became less dependent on frequency. This is because the induced charges gradually fail to follow the reversing field causing a reduction in the electronic oscillations as the frequency increased. The increase in dielectric loss with increasing filler contents may be attributed to the interfacial polarization mechanism of the heterogeneous system. Further moderate increase in $\tan \delta$ occurs due to irradiation. The growth in $\tan \delta$ and thus increase in conductivity is brought by an increase in the conduction of residual current and the conduction of absorption current (Bogoroditsky et al 1974).

\subsection{Conductivity of composite}

Figure 3(a, b) shows the a.c. conductivity of the pristine and irradiated composites as a function of frequency and concentration of the filler. The a.c. conductivity of the composites increased with increasing frequency and concentration of filler. It was also observed that the conductivity further increased upon ion beam irradiation. It is assumed that an electrical conducting path and network of connections could be formed in the composites with increasing content of the filler (figure 3(c)). It is known that the electrical conductivity of such composites depends on the type and

Table 1. \% Crystallinity and crystalline size of filler.

\begin{tabular}{lccccc}
\hline & \multicolumn{2}{c}{$\begin{array}{c}\text { Average crystalline } \\
\text { size }(\mathrm{nm})\end{array}$} & & \multicolumn{2}{c}{$\%$ Crystallinity } \\
\cline { 2 - 3 } \cline { 5 - 6 } $\begin{array}{l}\text { Filler } \\
\text { concentration } \\
(\text { wt.\%) }\end{array}$ & Pristine & $\begin{array}{c}5 \times 10^{12} \\
\text { ions } / \mathrm{cm}^{2}\end{array}$ & & Pristine & $\begin{array}{r}5 \times 10^{12} \\
\text { ions } / \mathrm{cm}^{2}\end{array}$ \\
\cline { 5 - 6 } Ni powder & 39.8 & - & & 20.8 & - \\
10 & 34.4 & 32.6 & & 15.6 & 11.6 \\
20 & 36.1 & 27.2 & & 19.9 & 16.7 \\
40 & 39.9 & 36.7 & 27.3 & 22.4 \\
\hline
\end{tabular}

concentration of the fillers (Abu-Abdeen et al 2002; Mamunya Ye et al 2002). Irradiation is expected to promote the metal to polymer bonding and convert the polymeric structure into a hydrogen depleted carbon network due to emission of hydrogen and/or other volatile gases. It is this carbon network that is believed to make the polymer more conductive (Wang et al 2004; Shah et al 2008).

\subsection{X-ray diffraction}

X-ray diffraction spectra as shown in figure 4(a) revealed the amorphous nature of pure PMMA and crystalline behaviour

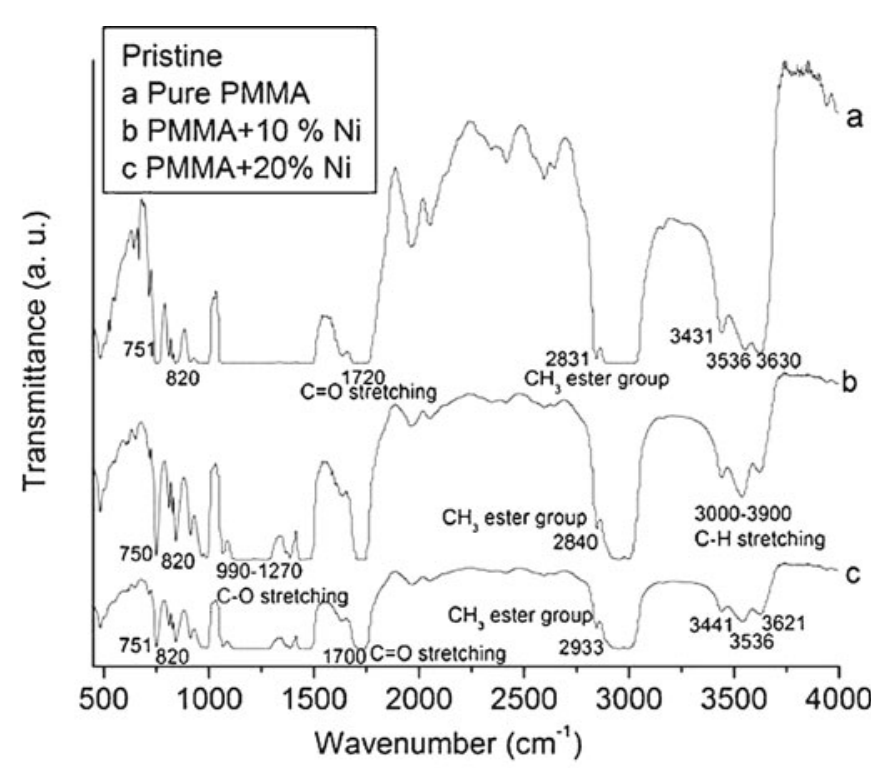

(a)

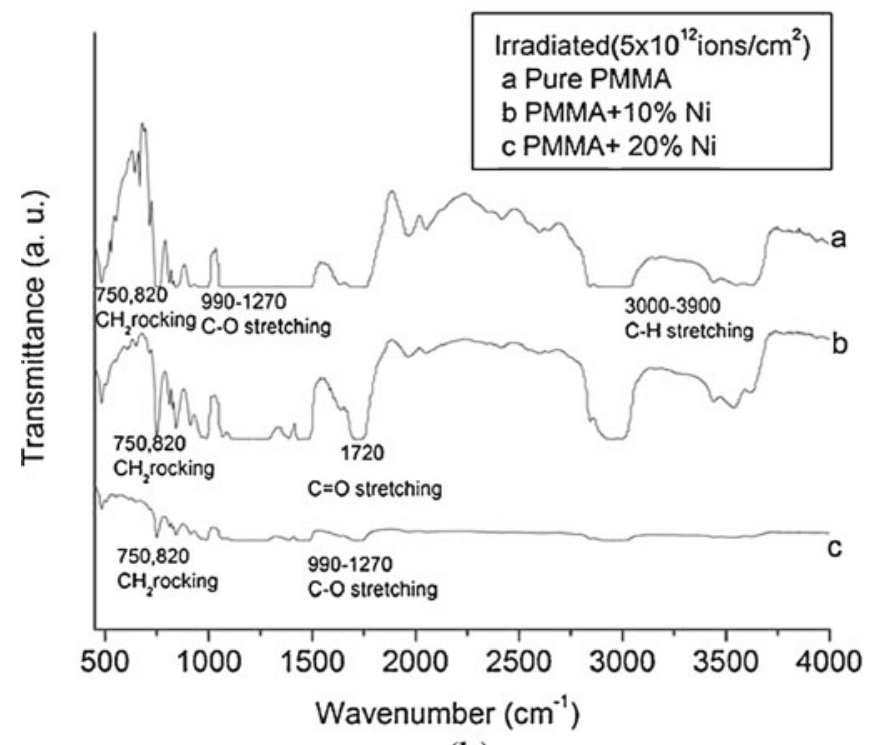

(b)

Figure 5. FTIR spectra of (a) pristine and (b) irradiated composite films. 
of Ni powder. The average particle size of the Ni powder obtained was $39.8 \mathrm{~nm}$. The most prominent peaks are obtained at $2 \theta \sim 51.9$ and 44.6 in all the cases as shown in figure 4 . The appearance of sharp peak in composite indicates some degree of crystallinity, although the decrease in intensity of the diffraction peak and a slight broadening of the peak after irradiation gives evidence of decrease in crystallinity. However, no considerable change in the peak position is observed, which reveals that the lattice parameters do not change significantly (Shah et al 2008). The crystallite size was calculated before and after irradiation using Scherrer's (1918) formula,

$$
b=K \lambda / L \cos \theta,
$$

where $b$ is FWHM in radians, $k$ the wavelength of X-ray beam $(1.5418 \AA), L$ the crystallite size in $\AA, K$ a constant which varies from 0.89 to 1.39 , but for most of the cases it is close to 1 . Percentage crystallinity of the composites was determined by area ratio method. In this method, the areas of amorphous and crystalline parts of the pattern were calculated (Kavlak et al 2003). The average crystallite size and \% crystallinity of the pristine and irradiated samples are listed in table 1. Irradiation induces large amount of energy deposition in the material and leads to decrease in crystallite size which may be attributed to splitting of crystalline grains. The irradiation may cause chain scissioning, which is also corroborated with FTIR spectra, assumed to be responsible for the reduction in crystallinity of the composite.

\subsection{Chemical response (FTIR spectroscopy)}

Chemical response or the nature of chemical modifications can be studied through the characterization of the vibration modes determined by infrared spectroscopy. Figure 5(a, b) represents the FTIR spectra of the pristine and irradiated (at fluence of $5 \times 10^{12}$ ions $/ \mathrm{cm}^{2}$ ) Ni-PMMA composites at different concentrations. It shows the interactions between macromolecules and filler particles and as a result, small shift and alteration of band shape depending on the immediate environment of the nearest surrounding of the functional groups. These observations are illustrated in figure 5 for bands in the wave number region of $500-4000 \mathrm{~cm}^{-1}$. For example, band at $2831 \mathrm{~cm}^{-1}$ in pure PMMA spectrum occurs at $2840 \mathrm{~cm}^{-1}$ for PMMA+ $10 \% \mathrm{Ni}$ and at $2933 \mathrm{~cm}^{-1}$ for
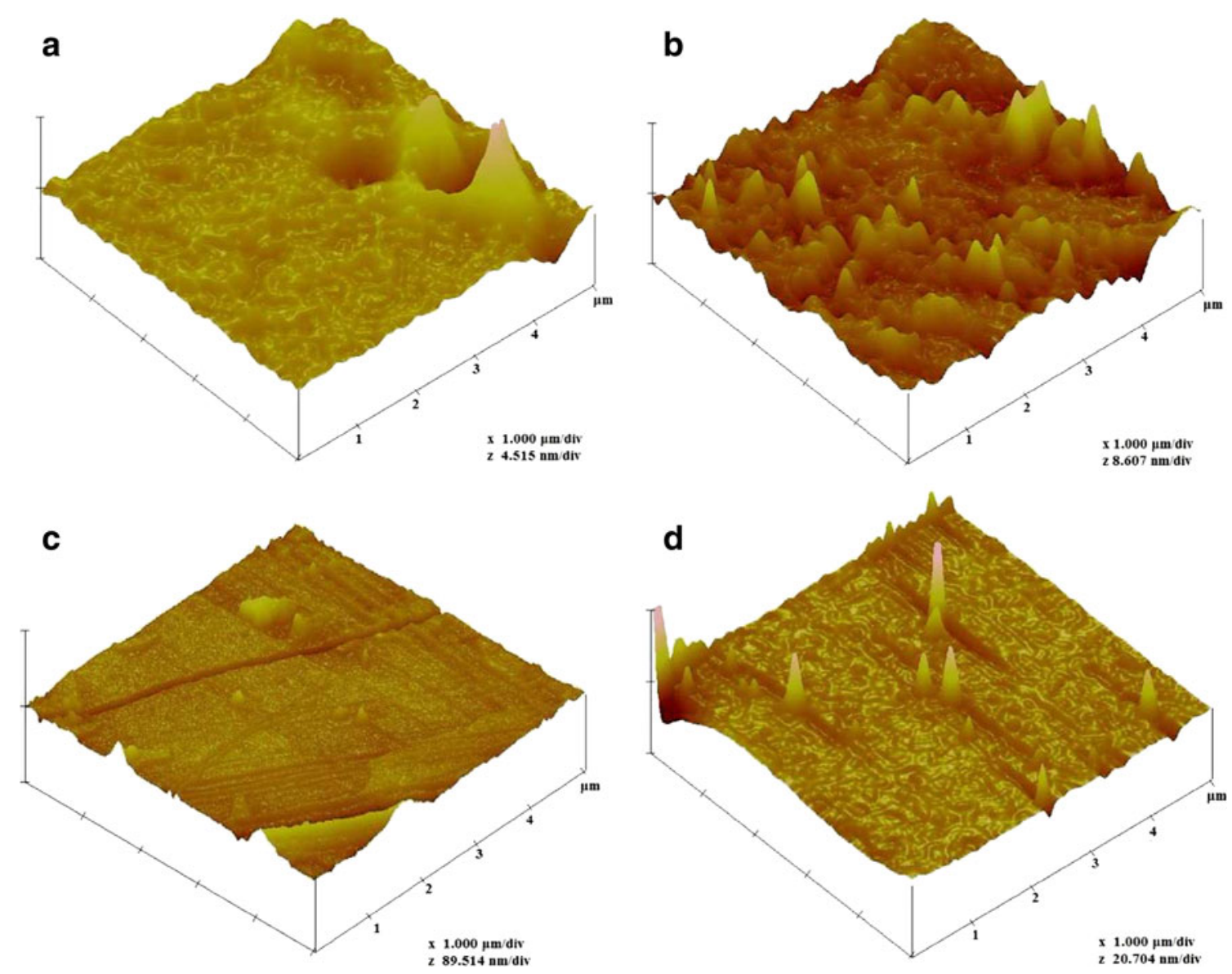

Figure 6. AFM images of (a) PMMA $+10 \% \mathrm{Ni}$ (pristine), (b) PMMA $+10 \% \mathrm{Ni}$ (irradiated), (c) $\mathrm{PMMA}+40 \% \mathrm{Ni}$ (pristine) and (d) PMMA+40\% Ni (irradiated) films. 

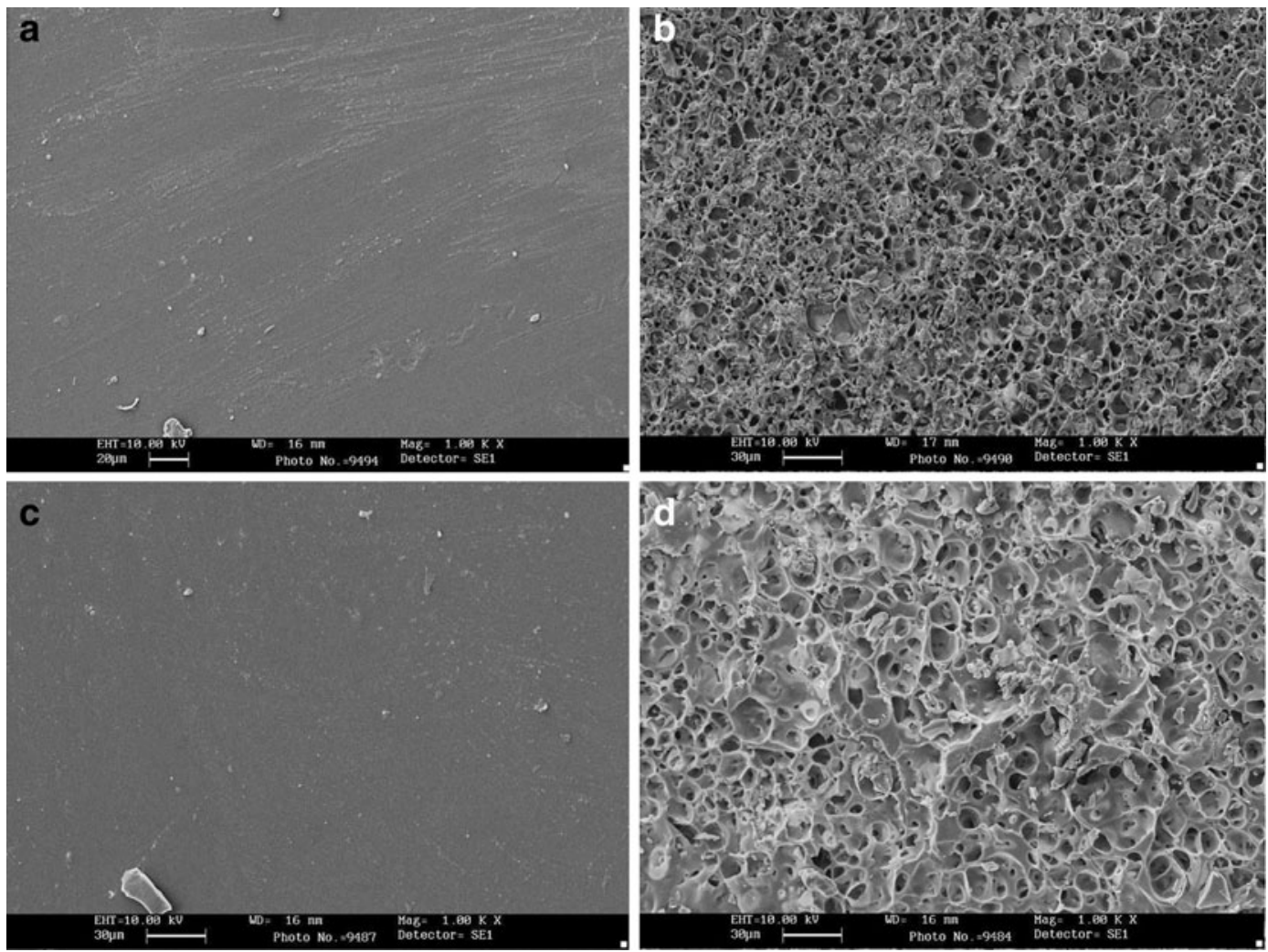

Figure 7. SEM images of (a) PMMA $+10 \% \mathrm{Ni}$ (pristine), (b) PMMA $+10 \% \mathrm{Ni}$ (irradiated), (c) PMMA $+40 \% \mathrm{Ni}$ (pristine) and (d) PMMA $+40 \% \mathrm{Ni}$ (irradiated) films.

PMMA $+20 \% \mathrm{Ni}$. The band in the region $1000-1700 \mathrm{~cm}^{-1}$, showed a few new peaks formed due to the presence of filler, and these peaks were not present in pure PMMA spectrum. Figure shows clearly the reduction in intensity of the peaks of irradiated samples as compared to pristine sample. A peak at $1720 \mathrm{~cm}^{-1}$ is identified as $\mathrm{C}=\mathrm{O}$ stretching vibrations in the pendant group $\left(-\mathrm{COOCH}_{3}\right)$ of PMMA. This peak decreased upon irradiation. An absorption band in the range of $1500-700 \mathrm{~cm}^{-1}$ comes from the $\mathrm{C}-\mathrm{O}$ stretching vibration $\left(1270-990 \mathrm{~cm}^{-1}\right), \mathrm{C}-\mathrm{H}$ bending vibration (1450-1350 $\mathrm{cm}^{-1}$ ) and $\mathrm{CH}_{2}$ rocking vibration (810 and $750 \mathrm{~cm}^{-1}$ ) (Choi et al 2001). These peaks decreased due to irradiation. This reveals that the intensity of $\mathrm{C}-\mathrm{O}, \mathrm{C}=\mathrm{O}$ bonds and $\mathrm{CH}_{2}, \mathrm{CH}_{3}$ groups are reduced upon irradiation. It indicates that polymer structure is converted into hydrogen depleted carbon network due to emission of hydrogen and/or other volatile gases.

\subsection{Atomic force microscopy}

The surface morphology of pristine and irradiated films of $10 \%$ and $40 \% \mathrm{Ni}$ dispersed PMMA was studied by AFM on $5 \times 5 \mu \mathrm{m}^{2}$ area as shown in figure 6(a-d). Each AFM image was analysed in terms of surface average roughness $\left(R_{\mathrm{a}}\right)$. The average roughness values obtained for unirradiated films are $0.7 \mathrm{~nm}$ and $3.2 \mathrm{~nm}$ for PMMA+10\% Ni and PMMA $+40 \% \mathrm{Ni}$, respectively. However, these values increased to $5.2 \mathrm{~nm}$ and $12.3 \mathrm{~nm}$ after irradiation at a fluence of $5 \times 10^{12}$ ions $/ \mathrm{cm}^{2}$. It is found that roughness increases as filler $(\mathrm{Ni})$ concentration increases. The increase in roughness may be due to the increase of density and size of metal particles on the surfaces of the PMMA films (Yan et al 2004). Ion irradiation of polymers leads, in general, to an increase in surface roughness due to large sputtering effects, which is also corroborated with SEM results.

\subsection{SEM micrographs}

Figure 7(a-d) shows the SEM images of $10 \%$ and $40 \% \mathrm{Ni}$ powder dispersed in pristine and irradiated composite films with $1 \mathrm{kX}$ magnification. After irradiation, significant changes in surface morphology were observed. Considerable damage in the polymeric structure was observed after irradiation, which is also responsible for decrease in crystallinity of the material as indicated by XRD analysis.

\section{Conclusions}

The conductivity of the composites increased with an increase in frequency and the content of the filler. Further, ion beam irradiation significantly enhanced dielectric 
properties of composite. It may be attributed to (i) metal to polymer bonding and (ii) conversion of the polymeric structure into a hydrogen depleted carbon network. Thus irradiation made the polymer more conductive. The dielectric properties of pristine and irradiated PMMA composites were studied over a broad range of frequency and at different concentrations of filler. Dielectric loss and constant are observed to change significantly after irradiation. This might be attributed to the breakage of chemical bonds and resulting in an increase of free radicals, unsaturation etc as revealed from FTIR analysis. XRD result showed that crystalline size and percentage crystallinity decreased after irradiation and that revealed amorphization of the sample due to irradiation which is responsible for the change in electrical properties. Surface roughness was observed to increase after irradiation as revealed from AFM/SEM images.

\section{Acknowledgements}

The authors are thankful to operating staff of Pelletron, IUAC New Delhi, for technical help. Financial support given by IUAC New Delhi, is gratefully acknowledged. We are also thankful to Mr Narendrasinh Chauhan, FCIPT, IPR, for help in SEM studies.

\section{References}

Abu-Abdeen M, Nasr G M, Osman H M and Abound A I 2002 Egypt. J. Solids 25275

Bedell C J, Solfield C J, Bridwell L B and Brown I M $1990 \mathrm{~J}$. Appl. Phys. 671736

Blunk R H J, Lisi D J, Yoo Y E and Tucker III C L 2003 AIChE J. 4918

Bogoroditsky N P, Pasynkov V V and Tareev B M 1974 Electrical engineering materials (Moscow: Mir Publisher)

Campbell J A, Goodwin A A and Simon G P 2001 Polymer 424731

Choi H W, Woo H J, Hong W, Kim J K, Lee S K and Eum C H 2001 Appl. Surf. Sci. 169-170 433
Creighton C J and Clyne T W 2000 Compos. Sci. Technol. 60 525

Dang Z-M, Zhang Y-H and Tjong S C 2004 Synth. Met. 14679

Foulger S H 1999 J. Appl. Polym. Sci. 721573

Ghany S, Salam A E and Nasr G M 2000 J. Appl. Polym. Sci. 77 1816

Hnatowicz V, Kvitek J, Svorcik V and Rybka V 1994 Appl. Phys. A58 349

Jonscher A K 1983 Dielectric relaxation in solids (London: Chesla Dielectric Press)

Kavlak S, Can H K, Guner A and Rzaev Z 2003 J. Appl. Polym. Sci. 901708

Louis P and Gokhale A M 1996 Acta Mater. 441519

Luo Y, Wang G and Zhang B 1998 Eur. Polym. J. 341221

Mamunya Ye P, Davydenko V V, Pissis P and Lebedev E V 2002 Eur. Polym. J. 381887

McLachlan D S, Blaszkiewicz M and Newmann R E $1990 \mathrm{~J}$. Am. Ceram. Soc. 7392187

Qureshi A, Singh N L, Rakshit A K, Singh F, Avasthi D K and Ganesan V 2007 Surf. Coat. Technol. 2018225

Raja V, Sharma A K and Narasimha V V R 2004 Mater. Lett. 58324

Scherrer P 1918 Gott. Nachar 298

Shah S, Singh N L, Qureshi A, Singh D, Singh K P, Shrinet V and Tripathi A 2008 Nucl. Instrum. Meth. $\mathbf{B 2 6 6} 1768$

Shepard D D and Twombly B 1996 Thermochim. Acta 272125

Singh N L, Shah S, Qureshi A, Singh F, Avasthi D K, Shrinet V and Ganesan V 2008 Polym. Degrad. Stabil. 931088

Solfield C J, Sugden S, Ing J, Bridwell I B and Wang Y 1993 Vacuum 44285

Song Y and Zheng Q 2004 Polym. Int. 53517

Vacik J, Cervena J, Fink D, Klett R, Hnatowicz V, Popok V and Odzhaev V 1997 Rad. Eff. Def. Sol. 143139

Verma A, Saxena A K and Dube D C 2003 J. Magn. Magn. Mater. 263228

Wang Y Q, Curry M, Tavenner E, Dobson N and Giedd R E 2004 Nucl. Instrum. Meth. B219-220 798

Yan X, Tao X, Shan X, Xiaobo W and Shengrong Y 2004 Nanotechnology 151759

Yu D, Wu J, Zhou L, Xie D and Wu S 2000 Compos. Sci. Technol. 60499

Zhang B, Xie C, Hu J, Wang H and Hai G Y 2006 Compos. Sci. Technol. 661558 